\title{
ANÁLISIS DE DOS MODELOS DE GOBERNANZA: LOS SISTEMAS AGROALIMENTARIOS LOCALES DE ZARZAMORA EN MÉXICO Y DE MORA EN COLOMBIA
}

\author{
Gerardo Torres Salcido \\ Universidad Nacional Autónoma de México (UNAM). Centro de Investigaciones sobre América Latina y el Caribe (Ciudad de México) \\ tsalcido@unam.mx \\ ORCID ID: https://orcid.org/0000-0002-0482-8847
}

Adriana Sandoval Moreno

UNAM. Unidad Académica de Estudios Regionales (Jiquilpan, México) coraliaz@yahoo.com asandoval@humanidades.unam.mx ORCID ID: https://orcid.org/0000-0003-2061-3456

Nathaly Burbano Muñoz

Escuela Superior de Administración Pública (Nariño, Colombia)

nathaly.burbano@esap.edu.co

ORCID ID: https://orcid.org/0000-0003-2965-2793

Recibido: 21/01/2020; Aceptado: 17/08/2020

Cómo citar este artículo / citation: Torres Salcido, Gerardo; Sandoval Moreno, Adriana y Burbano Muñoz, Nathaly (2020). Análisis de dos modelos de gobernanza: los sistemas agroalimentarios locales de zarzamora en México y de mora en Colombia. Estudios Geográficos, 81 (289), e049. https://doi.org/10.3989/estgeogr.202063.063

RESUMEN: En este artículo se exponen los resultados de una investigación sobre la gobernanza de los sistemas agroalimentarios locales. Se estudian dos sistemas productivos: la zarzamora (Rubus fruticosus) en el municipio de Los Reyes, Michoacán, México; y la mora (Rubus glaucus Benth) en el municipio de Pasto, Nariño, Colombia. La metodología aplicada tiene un enfoque cualitativo y está basada en recorridos de campo, entrevistas informales y semiestructuradas, así como en dinámicas grupales. Este enfoque fue complementado con fuentes bibliográficas y estadísticas. Ambos casos de estudio son críticos o de contraste porque se analizan modelos de gobernanza que muestran las contradicciones de la economía agroexportadora, como en el caso de Los Reyes, y de la campesina, en el caso de Pasto, en torno a un producto agrícola con alta demanda. La hipótesis de investigación es que la gobernanza de ambos sistemas se realiza de manera jerárquica, con el papel protagónico del mercado de exportación, en un caso, o de la gestión pública, en el otro. Sin embargo, en los intersticios de estos tipos de coordinación se mueven los actores que permiten observar la construcción incipiente de formas de gobernanza descentralizada y de sistemas agroalimentarios locales, con base en la apropiación de las tecnologías de cultivo, la transmisión del conocimiento y el arraigo territorial del producto. Se concluye que la investigación de estos modelos de gobernanza es de importancia para las políticas públicas de desarrollo territorial.

PALABRAS CLAVE: gobernanza territorial, zarzamora, mora, agroindustria, desarrollo local, Michoacán, Nariño.

\section{ANALYSIS OF TWO MODELS OF GOVERNANCE: LOCAL AGRO-FOOD SYSTEMS OF BLACKBERRY IN MEXICO AND COLOMBIA}

ABSTRACT: This article presents the results of a research on governance of local agro-food systems. Two production systems are studied: the blackberry (Rubus fruticosus) in the municipality of Los Reyes, Michoacán, México; and mora or also blackberry (Rubus glaucus Benth) in the municipality of Pasto, Nariño, Colombia. The applied methodology is based on a qualitative approach: field trips, direct observations, informal and semi-structured interviews complemented with bibliography and statistical sources. By drawing in contrast techniches governance models are analyzed in order to show the contradictions of the agro-export economy: in the case of Los Reyes, with respect to the peasant one, and in the case of Pasto around the blackberry. The research hypothesis is that the governance of both systems is carried out hierarchically, with the leading role of the export market, in one case or of the public management, in the other. However, in the interstices of these types of coordination there are movements of stakeholders which allow to observe an incipient construction of decentralized governance and local agro-food systems based on the appropriation of agriculture technologies, transmission of knowledge and territorial anchorage of the product. It is concluded that the research of these governance models is relevant for the policies of territorial development.

KEYWORDS: territorial governance, blackberry, agro-industry, local development, Michoacán, Nariño.

Copyright: ( 2020 CSIC. Este es un artículo de acceso abierto distribuido bajo los términos de la licencia de uso y distribución Creative Commons Reconocimiento 4.0 Internacional (CC BY 4.0). 


\section{INTRODUCCIÓN}

La gobernanza territorial se ha adoptado por su potencial analítico de las formas de coordinación y de los acuerdos multinivel que establecen los actores locales. Una definición muy extendida hace referencia a los procesos sociales y políticos mediante los cuales se deciden los objetivos de convivencia en torno a temas de interés colectivo (Aguilar, 2006), timoneados por la administración pública (Porras 2019). Si bien esta definición se refiere al papel de los distintos niveles de gobierno para lograr las metas de la sociedad, en los ámbitos rurales la gobernanza es más compleja, pues se encuentra vinculada a la construcción de sistemas agroalimentarios locales (SIAL), definidos como una red de productores agrícolas, empresas e instituciones especializadas en la producción y promoción de productos con identidad territorial (Sanz y Muchnik, 2016).

Torre y Traversac (2011) consideran que la la construcción de objetivos comunes en los sistemas agroalimentarios requiere el conocimiento de las regulaciones, normas y reglamentos, tanto de las organizaciones internacionales como de los distintos niveles de gobierno. Esto implica el análisis de los mecanismos de información y rendición de cuentas, reuniones y acuerdos vinculantes con los actores interesados. Para ello es muy importante construir lazos de confianza (Torres y Ramos, 2008) y conocer las dimensiones que adquieren los mecanismos de coordinación, mediante el análisis y comparación de casos (Torres y Sanz, 2018). De acuerdo con Kooiman (2003) es posible establecer tres tipos de gobernanza: la autogobernanza de las comunidades, la co-gobernanza entre el gobierno y actores poderosos, y la gobernanza jerárquica, que se define como una intervención externa cuyo objetivo es asignar funciones a los actores socio-políticos y a la naturaleza, en el caso de la explotación de los recursos.

Este último tipo de gobernanza es pertinente para analizar los modelos de coordinación de los sistemas productivos de la zarzamora (Rubus fruticosus) en el municipio de Los Reyes, en Michoacán, México, y la mora (Rubus glaucus Benth), en en el municipio de Pasto, Nariño, Colombia ${ }^{1}$. Ambos sistemas están enmarcados en la producción de berries o frutillas, como la fresa, el arándano o la frambuesa, en torno a las cuales se han desarrollado cadenas globales de valor (González,Santoyo, Arana y Muñoz, 2020), caracterizadas por el ordenamiento de las fases de producción, distribución y consumo a cargo de grandes com- pañías multinacionales. Debido a ello, las frutillas dejaron de ser productos tradicionales y se convirtieron en mercancías con alto valor en el mercado, por estar asociadas a dietas sanas y a representaciones de una vida saludable (Sánchez, 2008; Moreno, Villareal, Lagos, Ordoñez y Criollo, 2011; Sistema de Información de Precios y Abastecimiento del Sector Agropecuario, SIPSA, 2013; Daigle, Michelle, Ranco y Emery, 2019; Schmeda, Jiménez, Theodoluz y Ladio, 2019). Como ejemplo de su importancia económica, cabe subrayar que la superficie cosechada y la producción de berries experimentaron, a nivel mundial, tasas de crecimiento anual de $2,68 \%$ y $3,4 \%$ respectivamente, entre los años 2000 y 2017 (Food and Agriculture Organization of the United Nations, FAOSTAT, 2019).

Es importante mencionar este marco general, porque en este período México se colocó en el quinto lugar en la producción mundial de frutillas, sólo después de China, los Estados Unidos de América (EUA), Rusia y Polonia (Fideicomisos Institucionales en Relación con la Agricultura, FIRA, 2016).

En este contexto, el cultivo de la zarzamora ha venido ganando importancia en México. En 2014, su producción representó el $23 \%$ del total de frutillas, sólo detrás de la fresa, que acumuló el 69\% (FIRA 2016). El municipio de Los Reyes, en Michoacán, ha sido el espacio en el cual se ha dado el crecimiento más importante del cultivo y exportación de zarzamora. En 1995, Muñoz y Juárez reportaron 80 ha cosechadas (citados en Thiébaut, 2011), pero en 2017 se contabilizaban más de 5000 ha (Servicio de Información Agroalimentaria y Pesquera, SIAP, 2020).

De acuerdo con la literatura, los factores que han favorecido el crecimiento de este cultivo en ese municipio son: 1) la creciente demanda en los EUA, Canadá, Europa y China, entre otros países; 2) las redes de comercialización generadas por las compañías agroexportadoras (Sánchez, 2008) y otros intermediarios; y 3) la disminución del papel del Estado en América Latina (Thiébaut, 2009).

En cuanto al primer factor, es destacable que la zarzamora puede llegar al consumidor final gracias a cadenas de frío organizadas por las grandes empresas agroexportadoras y otros intermediarios, que han aprovechado la buena infraestructura de los centros productivos y su cercanía con la frontera norte. Esto ha permitido, de acuerdo con el United States Department of Agriculture, USDA (2019), que México se haya convertido prácticamente en el único proveedor 
de los EUA durante el otoño e invierno de zarzamora fresca, con una participación de $97,8 \%$ en valor y en volumen.

Respecto al segundo factor, la distribución está dominada por tres tipos de intermediarios: el primero corresponde a las empresas multinacionales como Sun Belle, Driscoll's, Berry Farm, Fresh Kampo y la nacional Grupo Heres. El segundo, son los intermediarios conocidos como brokers, quienes operan como representantes de cadenas de supermercados con oficinas especializadas en la frontera norte, aunque también facilitan la exportación de zarzamora hacia Canadá, Europa y China (Sánchez, 2008; Ibarra, Romero, Jaime y Hurtado, 2013; Morales, Cantillo y Colín, 2019). El tercer tipo de intermediario es el que que compra la zarzamora rechazada por las empresas agroexportadoras por no reunir los requisitos de calidad establecidos, para distribuirla en la agroindustria nacional, las centrales de abasto y los mercados locales.

El último factor, el cambio de las funciones del Estado en la agricultura y en las áreas rurales de América Latina, tiene su origen en profundos cambios institucionales que se dieron a finales del Siglo XX. En México, la reforma de 1992 del artículo 27 constitucional dio fin al reparto agrario, permitiendo la venta o renta de tierras ejidales. Esta reforma se realizó en la perspectiva de la firma del Tratado de Libre Comercio de América del Norte (TLCAN), que entró en vigor en 1994. De acuerdo con Merchand (2013), esta situación fue aprovechada por las compañías trasnacionales en Los Reyes para impulsar el negocio de la agroexportación.

En Colombia, la Constitución de 1991 redefinió los términos de acceso a la tierra para grupos indígenas y afro-colombianos reconociendo la tenencia colectiva, como lo había hecho la Constitución de 1917 en México (Hoffmann y Agudelo, 1998), con la salvedad que conservó el latifundismo y dejó en la indefinición al campesinado, al que sólo menciona como trabajador agrario. Puede señalarse, no obstante, que a partir de la promulgación de la Constitución se ha planteado la posibilidad de integrar la agricultura familiar a cadenas de valor.

En este marco, aunque el sistema productivo de la mora se ha caracterizado por su vinculación a unidades campesinas y a sistemas pluricultivo, desde hace años ha sido considerado como una oportunidad para incursionar en los mercados globales. Para lograr ese objetivo se formularon, en años pasados, programas públicos de apoyo, mediante la distribución de semillas y plántulas híbridas para impulsar una mayor productividad (Tobasura y Ospina, 2011; Henao y Tobasura, 2018; Alwang et al., 2019).

Con relación a todos estos antecedentes, este artículo busca responder a la pregunta sobre cuáles son los modelos de coordinación y gestión de la agroindustria de la zarzamora y la producción campesina de la mora, así como sus efectos en la organización de los actores socioterritoriales. La hipótesis de trabajo inicial es que la coordinación de estos sistemas responde al tipo de mercado y a la intervención institucional, que como agentes externos, organizan jerárquicamente el territorio, lo que influye en las características del proceso productivo, la comercialización y el medio ambiente. El objetivo que persigue esta contribución es aportar conocimiento sobre sistemas agroalimentarios con grandes diferencias de tipo productivo y organizativo, sus interacciones multinivel y sus modos de gobernanza, aspectos insuficientemente tratados en la literatura de los SIAL (Giacomini y Mancini, 2015; Comisión Económica para la América Latina, CEPAL, 2016;Trueba, Infante y Ayala, 2018).

El artículo se estructura de la siguiente manera. En la sección 2 se abordan las fuentes y la metodología. Este apartado incluye la localización de los casos, los recursos bibliográficos y estadísticos consultados, la metodología, las entrevistas y talleres realizados en ambos territorios y las técnicas de recolección e interpretación de datos. En el tercer apartado, los resultados analizan tres dimensiones de la gobernanza construidas a partir de la investigación directa y de las informaciones aportadas por los actores: las características de los procesos productivos; las redes de comercialización y las tensiones ambientales. La sección 4 está dedicada a la discusión de los resultados y a las perspectivas del desarrollo en los casos estudiados. Se concluye con los desafíos que estos sistemas agroalimentarios locales enfrentan para construir formas de gobernanza distintas a la jerarquía impuesta por los agentes externos como alternativas al desarrollo territorial.

\section{FUENTES Y METODOLOGÍA}

\section{Localización de los casos}

El cultivo de zarzamora en México se encuentra altamente localizado en el municipio de Los Reyes y otros 
aledaños, como Tocumbo y Peribán. Según Sánchez (2008), su ubicación geográfica es muy favorable para el cultivo de la zarzamora. Su altitud varía entre los 900 y 3400 msnm. Debido a que se encuentra en el eje Neovolcánico, Los Reyes está protegido contra vientos fuertes y tiene una precipitación media anual de 1151 mm (Comisión Nacional del Agua, CONAGUA, s.f). El rango de temperatura es de 10 a $24^{\circ} \mathrm{C}$ con clima templado subhúmedo y lluvias en verano (Instituto Nacional de Estadística y Geografía, INEGI, 2009).

En Colombia, el cultivo de la mora se realiza principalmente en Cundinamarca, Santanderes, Antioquía, Huila, Caldas, Quindío, Risaralda, Tolimá, Valle, Cauca y Nariño. En 2017, el cultivo de esta frutilla sobrepasó las 13000 ha y Nariño participó con un 7,7\%. Por su parte, el municipio de Pasto tuvo 123 ha cosechadas. De acuerdo con el diagnóstico de Nariño, elaborado por el Consejo Nacional de la Política Económica y Social de la República de Colombia (Consejo Nacional de Política Económica y Social, CONPES, 2014), este de- partamento tiene condiciones propicias para el desarrollo de la cadena de valor de la mora. Se encuentra ubicado en el suroccidente de Colombia, entre la cordillera de los Andes y el Océano Pacífico. Limita al norte con el departamento del Cauca, al sur con la República del Ecuador, al oriente con el departamento de Putumayo y al occidente con el Océano Pacífico. Pasto se encuentra a una altitud media de $2527 \mathrm{msnm}$. Las temperaturas medias mínimas y máximas son de 6 a $11^{\circ} \mathrm{C}$ (Benavides,Tarlé y Galbiatti, 2009). La precipitación media anual está entre los 1000 y 1500 mm y predomina el clima semihúmedo (Gobierno de Colombia, 2020). Véase Figura 1.

\section{Metodología}

La metodología de esta investigación es de corte cualitativo. Se adopta el modelo de análisis de "casos críticos" (Giménez y Heau, 2014, p. 357; Flyvbjerg,

FIGURA 1.

LOCALIZACIÓN DE LOS CASOS DE ESTUDIO

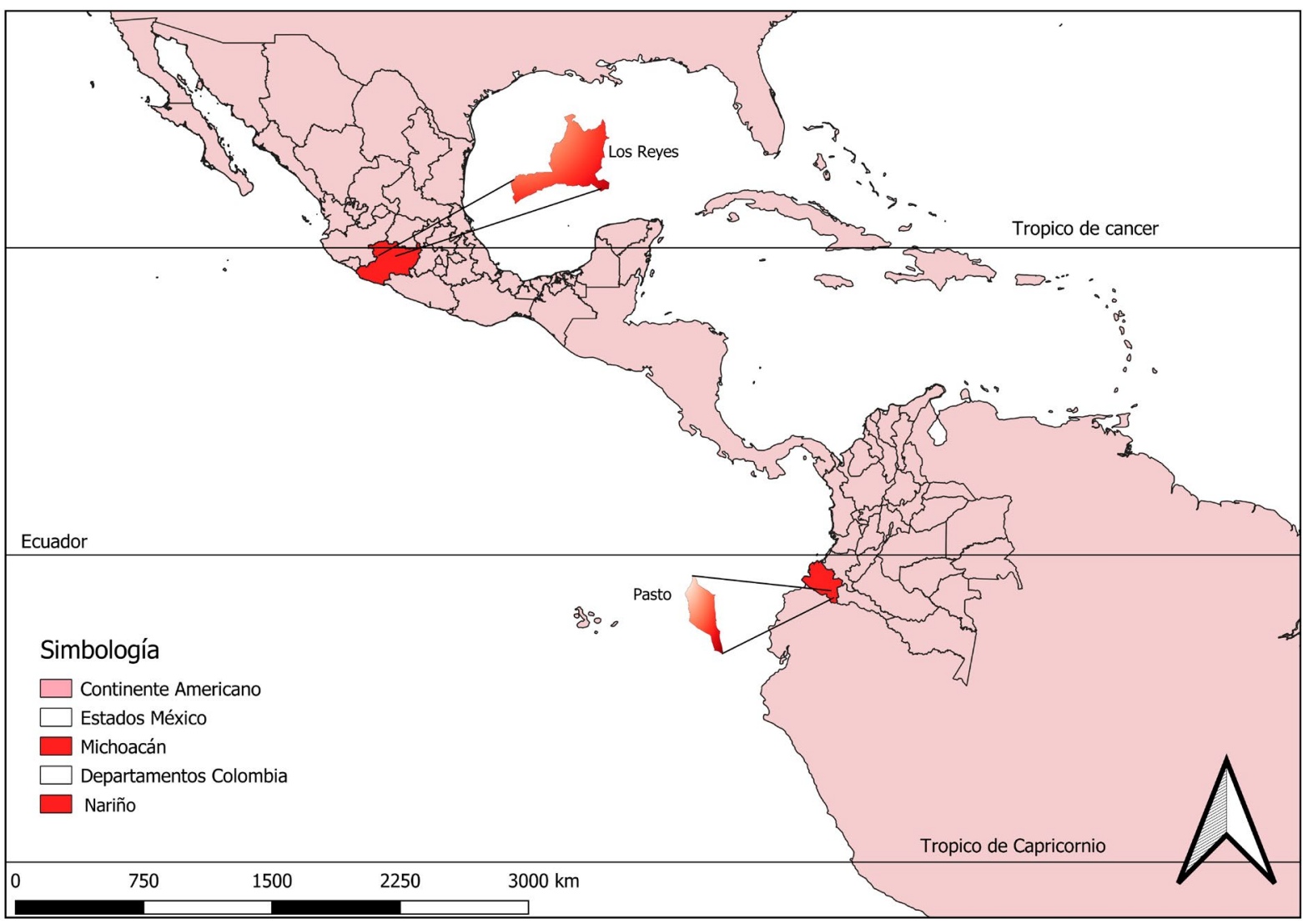

Fuente: Elaboración propia. 
2001) como ejemplo de dos situaciones de contraste, pero que pueden ser ejemplares de los procesos socioterritoriales. La estrategia metodológica se basa en observaciones directas, entrevistas semiestructuradas y talleres, complementada con estadísticas, informes y bibliografía sobre ambos casos². Las fuentes de información consultadas son las bases de datos del USDA y FAOSTAT, el SIAP de México y las bases del Departamento Administrativo Nacional de Estadística (DANE), así como Agronet, del Ministerio de Agricultura, en Colombia.

En cuanto a las fuentes bibliográficas, se revisó la literatura nacional e internacional y se recuperaron tesis y documentos en las áreas de estudio, lo que permitió formular hipótesis iniciales. En el trabajo de campo se llevaron a cabo recorridos de observación en las localidades, se conversó con agentes involucrados en la producción, comercialización y consumo de frutillas y se establecieron contactos con actores considerados clave y a quienes se aplicaron entrevistas semi estructuradas.

En el caso de Michoacán, se realizaron recorridos de campo visitando las parcelas, empacadoras y empresas exportadoras. Asimismo, se llevaron a cabo numerosas conversaciones con funcionarios de los tres niveles de gobierno, académicos, trabajadores de las empresas y jornaleros que culminaron en un taller de análisis de Fortalezas, Oportunidades, Debilidades y Amenazas (FODA) en el mes de noviembre de 2017, en el cual participaron 26 personas: 6 productores de zarzamora; 1 funcionario público del gobierno estatal; 6 trabajadores de huertos, 12 alumnos de la Universidad de la Ciénega del Estado de Michoacán, así como una académica de la Universidad Nacional Autónoma de México (UNAM), quien fungió como coordinadora.

En Colombia, se llevó a cabo un trabajo de campo en Pasto, consistente en la recopilación de material documental, tesis, programas de gobierno y estadísticas municipales, recorridos de campo y conversaciones informales, así como el levantamiento de 10 entrevistas (funcionarios, académicos de la Universidad de Nariño, productores y consultores) durante el mes de junio de ese mismo año.

Tanto el taller como las entrevistas dieron la pauta para caracterizar el proceso productivo local y la coordinación de los actores, la comercialización y las tensiones medio ambientales, así como el papel de la gestión pública. En el siguiente apartado damos a conocer los resultados más relevantes.

\section{RESULTADOS}

\section{Características del proceso productivo}

El sistema agroalimentario de la zarzamora en México tiene un alto grado de localización territorial y de aglomeración empresarial en Los Reyes y el centro-occidente mexicano, por lo cual ha sido considerado un clúster (Sánchez, 2008) con vinculaciones fuertes al exterior y lazos débiles en el territorio, pues siempre existe el riesgo de deslocalizar la producción hacia otros lugares que cuenten con las condiciones para el cultivo de la frutilla.

Durante el año 2017, el municipio de Los Reyes tuvo 5060 ha de superficie cosechada (40\% de la superficie cosechada a nivel nacional). La producción, durante ese mismo año, fue de 131465 t (el 48,6\% de la producción nacional) y la productividad fue 26 t/ha, contra $13 \mathrm{t} /$ ha en promedio durante los años 2013-2015 (SIAP, 2020).

Para los participantes en el taller FODA mencionado anteriormente, este crecimiento extraordinario de la productividad es una fortaleza del territorio. Otras fortalezas relativas identificadas fueron: a) la introducción de variedades híbridas que han mejorado el tamaño, sabor y resistencia de la fruta; b) la técnica de producción forzada; c ) la especialización de la mano de obra para la recolección y poscosecha del producto; y d) el amplio margen de utilidad por hectárea cosechada. Algunas de estas fortalezas son también consideradas como oportunidades y amenazas, como veremos más adelante.

En cuanto a la introducción de nuevas variedades, para muchos pequeños productores representa una oportunidad, cuando se puede acceder a ellas sin erogar los costos asociados al uso de las patentes. Por ejemplo, la primera variedad introducida en Los Reyes fue la Brazos y actualmente predomina la Tupy, la cual ha sido liberada por las agroexportadoras y cuya ventaja consiste en requerir menos horas de frío. Sin embargo, otras variedades como la Dasha, Vanesa, Catherine y BQ patentadas por Driscoll's, son consideradas como una amenaza por la inversión que requieren y porque están desplazando a la Tupy de los mercados de exportación. Según Aguilar (2018), las variedades patentadas necesitan una inversión inicial de aproximada de 50000 US Dollars por ha, que representa cinco veces más de la inversión en la Tupy por hectárea no tecnificada ${ }^{3}$.

La técnica de la producción forzada, que los participantes consideran una aportación tecnológica de 
los productores de Los Reyes, consiste en la poda temprana de la planta (a los 5 meses), para detener su crecimiento e iniciar la maduración. Ésta se logra mediante la aplicación de productos químicos defoliantes. No obstante, esta técnica presenta amenazas ambientales porque los esquilmos son abandonados en los linderos de los huertos, con lo que se propicia la proliferación de plagas.

La existencia de una abundante mano de obra, sobre todo femenina, especializada en la pisca o recolección de la fruta ha sido central tanto en la productividad como en la conservación de las características sensoriales del fruto: "La mayoría de piscadores son mujeres, porque son las que piscan mejor, ya que tienen [..] sensibilidad con la fruta" (Mario, cajero y jornalero, comunicación personal, 22 de noviembre, 2017).

La demanda y el precio de la zarzamora, por otra parte, han tenido en los años recientes un crecimiento constante a nivel nacional. El precio medio rural de la tonelada en 2017 fue de 1800 US Dollars. Los productores de Los Reyes participaron con el 54\% del valor de la producción a nivel nacional, que ascendió a más de 500 millones de US Dollars (SIAP, 2020). No obstante, aunque esta participación en la superficie cosechada, volumen y valor es una fortaleza, también se ve como una amenaza en el mediano y largo plazo, pues existe el riesgo de que el mercado se desplome o se multipliquen los territorios productivos $y$, por tanto, los competidores.

El alto margen de utilidad del cultivo ha generado impactos en la renta de tierras por la incorporación de nuevos inversionistas. En 2017, el 60\% de los productores rentaba la tierra y habían sido atraídos recientemente por el negocio de la zarzamora, pues sólo tenían entre 1 y 3 años en él (Y. Morales, Universidad Michoacana de San Nicolás Hidalgo, comunicación personal, 27 de mayo, 2020). La razón es que “..en Michoacán una inversión inicial de 200 mil pesos (aprox. 10000 US Dollars) el primer y segundo año tienen un margen neto de utilidad cada uno de 400 mil pesos (20000 US Dollars) y el tercer año de 350 mil (17500 US Dollars)" (Aguilar, 2018), porque no se invierte en macrotúneles, pozos tecnificados ni en la sustitución de plántulas.

Si bien la especulación es una amenaza para los pequeños inversionistas, no es así para los ejidatarios quienes definen estrategias para compensar los peligros, ofreciendo en renta parte de sus tierras para obtener un ingreso seguro (Thiébaut, 2009) Las modificaciones constitucionales de 1992 y el TLCAN (sus- tituido en 2020 por el nuevo Tratado México, EUA y Canadá, T-MEC) propiciaron el mercado de renta de tierras, pero no se ha dado una gran concentración de la propiedad. La mayor parte de los productores poseen (en propiedad o renta) hasta 2,5 ha aunque se estima que aproximadamente el $10 \%$ tienen entre 16 y 23,5 ha. La zarzamora genera 5 empleos permanentes por hectárea, independientemente de los trabajadores eventuales que son contratados durante la pisca o recolección del fruto entre los meses de octubre y mayo (Servicios Informativos y Publicitarios del Sureste Mexicano, SIPSE, 2014).

Los riesgos generales que perciben los actores participantes en el taller para el sistema productivo de la zarzamora son también el monocultivo, la deforestación, la intermediación de las agroexportadoras y de otros canales de comercialización nacionales, así como la dependencia de un solo mercado, en este caso, de los EUA, aunque se está tratando de diversificar las exportaciones hacia Europa y China.

En el caso de Colombia, de acuerdo con el Ministerio de Agricultura, entre 2013 y 2017, el área cosechada de mora tuvo un crecimiento de $9,75 \%$ al pasar de 11998 ha a 13168 ha. La producción se incrementó un $17 \%$, al pasar de 105000 t a 123000 t en términos redondos, en tanto que la productividad creció en el mismo periodo de 8,78 a 9,35 t/ha. Nariño participó con un 5,8\% de la producción colombiana (DANE, 2019; Ministerio de Agricultura, Agronet, 2020). En este departamento, el área sembrada se ubica en los municipios de la zona sur oriental: Contadero, Ipiales, Pasto, Puerres, Sandoná, San Pablo y San Pedro de Cartago. En Pasto, el cultivo de mora se realiza en aproximadamente 123 ha en las que laboran entre 200 y 600 productores (Giovani G. Secretaría de Agricultura de Nariño, comunicación personal, 22 de junio, 2017). El cultivo se ubica fundamentalmente en las veredas (unidades político territoriales de carácter sub municipal en las áreas rurales o micro territorios en áreas urbanas) de Santa Clara y Santa Rosa (Edy O., Secretaría de Agricultura de Nariño, comunicación personal, 20 de junio, 2017).

En cuanto a las variedades de la fruta, si bien predominan las semillas híbridas, no hay variedades dulces, como las que se cultivan en Los Reyes. Son frutillas cuya acidez es característica y se conocen con distintos nombres, como de Castilla, la "Sin Espinas", Ranchona, Hartona, etcétera.

Una particularidad que resalta del cultivo de la mora en Pasto, es su asociación a la pequeña propie- 
dad y a la tenencia colectiva de la tierra. El predio campesino promedio es menor a 5 ha que está muy por debajo de la Unidad Agrícola Familiar (UAF) en Nariño, que es de 14 ha. El cultivo de la mora también se realiza en los resguardos indígenas, bajo un régimen colectivo de propiedad. Todo lo anterior contrasta con la gran concentración de la propiedad agraria. El $86,5 \%$ de los predios tiene menos de 10 ha y representan apenas el 31\%, mientras que el $0,7 \%$ concentran el $29 \%$ del área cultivable.

Otra característica, de acuerdo con las entrevistas realizadas en campo, ha sido el vacilante desempeño de la gestión pública. Los productores de mora tuvieron recursos gubernamentales significativos hasta 2014, dirigidos básicamente a la distribución de semillas e insumos. Pero, en 2017, dichos recursos habían descendido por la falta de apoyo para mejorar las condiciones de comercialización, manejo poscosecha y transformación agroindustrial del fruto.

Una característica más del sistema productivo de la mora en Pasto, como la de muchos otros productos tradicionales, es que se encuentra inmerso en sistemas que combinan diversos cultivos con la crianza de especies menores. En la unidad campesina familiar de pequeña extensión se intercalan los productos hortofrutícolas tradicionales de la zona (como la cebolla, la papa, la mora y las plantas medicinales, entre otros) con la crianza de cuyes y conejos. En este sentido, la mora responde no sólo a la dinámica de la ganancia, sino a estrategias de la unidad campesina enfocadas a su seguridad alimentaria y a la comercialización de excedentes que aportan ingresos permanentes.

El empleo que genera el cultivo de la frutilla es muy importante para mantener la unidad agrícola familiar. Según el Ministerio de Agricultura (2015), la mora genera 3,4 empleos directos e indirectos por hectárea, que es superior a los que crean los frutales. No obstante, de acuerdo con las entrevistas en campo, en el municipio de Pasto la mora genera hasta 6 empleos, aunque la superficie de cultivo no sobrepasa la media hectárea.

La dispersión y heterogeneidad del cultivo, su atomización (como señala uno de los entrevistados), su carácter complementario para las familias campesinas y otros problemas señalados por los actores, como el escaso procesamiento, la falta de apoyo para las cadenas de frío y las dificultades del transporte, han determinado que la mora se comercialice con gran ventaja para los intermediarios.
Las políticas de apoyo no han superado estos problemas, pues se limitan a la distribución de semillas y plántulas entre los campesinos y en los resguardos indígenas (Enrique C., dirigente de Asociación Hortofrutícola de Colombia, ASOHOFRUCOL, comunicación personal, 21 de junio, 2017), sin ofrecer un seguimiento técnico ni generar propuestas para valorizar el producto, a pesar de que los diagnósticos de políticas insisten en la urgencia de implementar programas incluyentes que mejoren las condiciones de vida de esos grupos de la población (Programa de las Naciones Unidas para el Desarrollo, PNUD, s.f).

\section{Coordinación multinivel y comercialización}

La coordinación de los actores en los procesos de comercialización está determinada, en primer lugar, por las características físicas y sensoriales de las frutillas y, en segundo término, por la cercanía o lejanía institucional y geográfica de los centros de consumo. Las frutillas no soportan la exposición al sol después de su cosecha, se deshidratan rápidamente y son susceptibles, por su alto contenido en agua, al ataque de hongos. Por ello, la calidad e inocuidad demandadas por los mercados internacionales y nacionales, influyen en los procesos de coordinación territorial de los actores en diversas escalas.

En Los Reyes, las redes de comercialización (Morales y Cantillo 2017) se han formado en torno a una gobernanza jerárquica para satisfacer la demanda de los centros de consumo de América del Norte. En la base de este sistema vertical, se encuentran los trabajadores especializados en el cultivo, cuidado, poda de la planta, en la recolección y empaque del fruto. Muchos de ellos tienen un trabajo permanente en la finca desempeñando diversas funciones "... casi son como 6 o 7 meses de trabajo, nomás en temporada de lluvia no se trabaja, que ya no hay fruta ni nada, solo trabajas levantando mata, mochándola, encuadrando varias cosas así. Solo en tiempos de lluvias cuando no hay fruta" (jornalero, comunicación personal, 22 de noviembre, 2017); “..de hecho, empecé a entrar nomás como ayudante, como acomedida, viendo y ya de ahí para delante empecé a manejar y aquí estamos [en el área de empaque]" (Guadalupe, empacadora y jornalera, comunicación personal, 13 de abril, 2018).

Un poco más arriba de esta base social se encuentran los productores individuales y organizados en sociedades y asociaciones. Entre los productores, se 
encuentran quienes sólo cultivan 2,5 ha o menos y aquellos que pueden concentrar una mayor cantidad de tierra (20 o más ha) con mayor capital para invertir en las variedades más rentables y cumplir con los estrictos procesos de control de calidad.

Después, se encuentran los prestadores de servicios; las instituciones educativas; los proveedores de crédito; los productores y vendedores de plantas, insumos y maquinaria; las empacadoras y otros agentes.

Los prestadores de servicios profesionales generalmente son extensionistas o personal con algún conocimiento técnico, a menudo empleados por las compañías de insumos o agroexportadoras. Se les denomina "ingenieros asesores". Son personajes clave para la venta de insumos ligados a los paquetes tecnológicos y para la gobernanza del sistema, pues como intermediarios vinculan a los productores con las agroexportadoras, las empresas de agroquímicos $\mathrm{y}$ las instituciones locales y federales.

Las instituciones de investigación y docencia, (universidades y escuelas tecnológicas regionales, así como las instituciones educativas nacionales con presencia en el estado de Michoacán), facilitan la formación de recursos humanos y la transmisión de conocimientos, pero su participación en los procesos de innovación es muy limitada, pues las grandes empresas como Driscoll's tienen sus propios departamentos de desarrollo tecnológico y contratos con las universidades de los EUA para desarrollar sus propias variedades y sistemas de innovación.

Los prestamistas, que desempeñan funciones de intermediarios, se aprovechan de la escasa presencia de los servicios financieros institucionales, tanto de la banca pública como la privada. La presencia de este agente en el territorio es una debilidad advertida por los actores del sistema productivo local, pues crea desigualdades por la concentración de las utilidades en los intermediarios y propicia la dependencia de los productores.

Todos estos actores se encuentran coordinados, como ya se ha mencionado, por las empresas agroexportadoras que ordenan el territorio orientándolo hacia los mercados externos, pero también por las regulaciones de los gobiernos estatal y federal (Sandoval, 2016; Sandoval, 2019). En la cúspide de esta gobernanza jerárquica se encuentran los supermercados y distribuidoras de EUA Canadá, Europa y Asia.

Las empresas agroexportadoras tienen la función de organizar el proceso productivo y asegurar la co- mercialización atendiendo a las exigencias de las buenas prácticas agrícolas, que van desde la producción, con instalaciones sanitarias dentro de los huertos, hasta el consumo, con la conservación del producto en frío y el buen manejo hasta su adquisición por el comprador final. Así, inmediatamente después del corte, la fruta se va colocando en empaques de PET (clamshells) de 125, 300 y 500 g, para ser transportados hasta los centros de exportación. El eje transversal del proceso es el cuidado de las características sensoriales de la fruta, es decir, evitar daños derivados de la recolección y poscosecha, así como de la transportación a las plantas enfriadoras $y$ empacadoras.

Las agroexportadoras más importantes se agrupan en la Asociación Nacional de Exportadores de Berries (ANEBERRIES), un grupo de presión constituido para incidir en las políticas de inocuidad y calidad implementadas por los gobiernos de los EUA y de México. En general, las organizaciones locales están excluidas de las decisiones sobre la calidad. Entre ellas, pueden mencionarse el gobierno municipal, las autoridades ejidales, las organizaciones de pequeños productores (ejemplos: Asociación de Productores de Zarzamora del Valle de Los Reyes, Asociación de Productores Agropecuarios por la Calidad, Unión de Productores de Zarzamora Los Reyes) y las pequeñas empresas empacadoras y procesadoras. Otras organizaciones tienen reconocimiento oficial, pero encuentran muchas dificultades para expresar los intereses de sus agremiados, como el Comité Estatal del Sistema Producto de la Zarzamora.

El aumento y dominio territorial de las empresas agroexportadoras es indudable, como lo refiere un ingeniero asesor: “... ya en estas fechas tenemos 29 exportadoras, hace 15 años teníamos dos, estas exportadoras de alguna manera han generado todo un desarrollo de crecimiento del cultivo. Como unas 9 o 10 son nacionales, las demás son mezclas de capitales chilenos, mexicanos, estadounidense o de capitales compartidos Chile y Estados Unidos" (Gabriel, ingeniero y productor, comunicación personal, 22 de noviembre, 2017).

El proceso de certificación de la calidad e inocuidad impone la intervención de las oficinas gubernamentales de EUA y México. Las primeras, expiden los permisos de sanidad vegetal, libre de plagas, por medio de la Fruits and Vegetables Import Requirements (FAVIR) del Servicio de Inspección de Sanidad Animal y Vegetal, del USDA. 
Las instituciones mexicanas que garantizan la vigilancia y regulación son la Secretaría de Agricultura, Ganadería, Pesca y Alimentación (SAGARPA, ahora Secretaría de Desarrollo Rural, SADER) y la Secretaría de Desarrollo Rural y Agroalimentario del estado de Michoacán. El Servicio Nacional de Sanidad, Inocuidad y Calidad Agroalimentaria (SENASICA), dependiente de la SADER, atiende las medidas de certificación fitosanitaria para productos de exportación establecidos por las Organizaciones Nacionales de Protección Fitosanitaria (ONPF), por lo cual está autorizada para expedir la lista de huertos certificados con buenas prácticas para la exportación (SENASICA 2019). La figura 2 muestra las interacciones a diversos niveles de los actores del sistema productivo en Los Reyes.

No obstante, en el control productivo y de propiedad intelectual que ejercen las agroexportadoras, destacan dos hechos que muestran un territorio en transformación: el aprovechamiento de canales nacionales, regionales y locales de comercialización, por un lado, y el surgimiento de agentes sociales innovadores.

Cuando la fruta fresca no reúne los requisitos de calidad exigidos por las exportadoras, por ejemplo, el color, tamaño, contenido de humedad o porcentaje de fruta dañada, emergen como protagonistas los canales de comercialización nacionales debido a una

FIGURA 2

INTERACCIONES MULTINIVEL EN EL SISTEMA PRODUCTIVO DE ZARZAMORA, MÉXICO

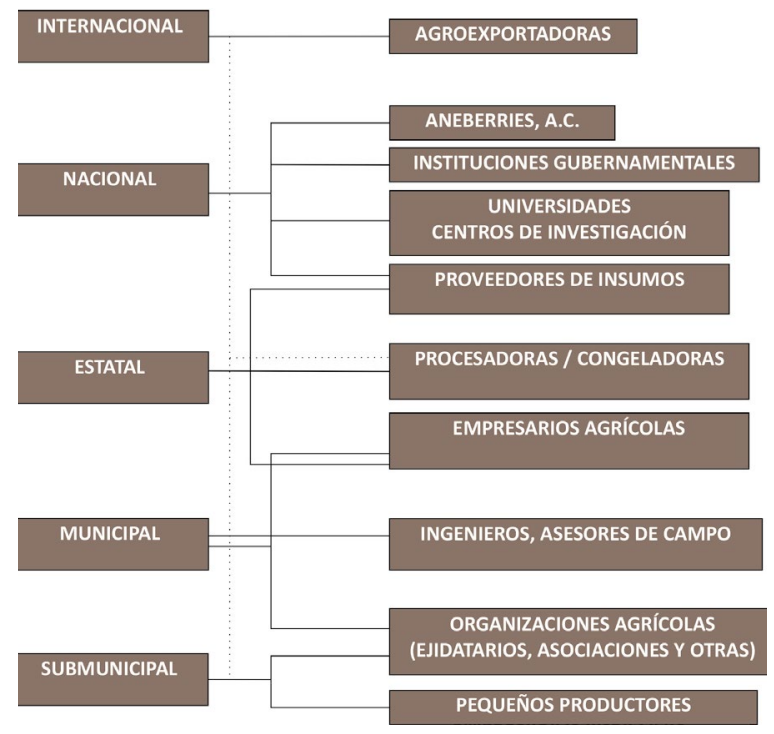

Fuente: Elaboración propia. creciente demanda en los mercados locales, pero con desventajas para los productores y con merma de calidad para los consumidores. Entre la zarzamora de segunda que rechazan las agroexportadoras, se encuentran las variedades liberadas y de acceso libre, como la Tupy. La caja de $1,5 \mathrm{~kg}$ de esta variedad se comercializa de 3,5 a 6 US Dollars, contra las variedades patentadas, que alcanzan precios de 24 a 36 US Dollars en los mercados internacionales (Aguilar, 2018).

La fruta rechazada por las agroexportadoras se destina principalmente a las industrias de procesamiento para elaboración de yogures, jugos (zumos), licores, repostería y panadería. Otra porción se canaliza en fresco a los mercados y supermercados nacionales, mercados municipales, restaurantes y pequeñas industrias familiares, así como a las tiendas locales e incluso se vende a pie de carretera.

Por otra parte, es reconocido que en Los Reyes existen agentes innovadores territoriales que han difundido mejoras en los procesos. Son productores experimentados que han logrado reconocimiento por sus labores de difusión, investigación, mejoramiento de los productos o la comercialización. Morales y Cantillo (2017) encuentran cuatro familias ubicadas por los productores como innovadoras. En este rubro también se encuentran los investigadores que trabajan en las empresas (Infante y Trueba, 2014) y otros profesionistas (ingenieros, administradores, capataces, etcétera), quienes han adquirido experiencia por medio del trabajo y han podido pasar de jornaleros a mayordomos o asesores, a encargados de huerta, capacitadores, supervisores e incluso, como buscadores de nuevas tierras que cumplan con las condiciones ambientales, accesibilidad vial y mano de obra para ampliar los cultivos.

Las políticas públicas también han desempeñado un papel importante en la innovación del sistema agroalimentario. El Plan de Desarrollo Integral del Estado de Michoacán 2015-2021, establece como una de sus prioridades "consolidar al sector agroalimentario de manera sustentable". En esta perspectiva se creó el Centro de Innovación y Desarrollo Agroalimentario de Michoacán A.C. (CIDAM), el cual tiene como misión participar en las cadenas agroalimentarias para elevar su competitividad técnica y económica (CIDAM, 2020)

Todo lo anterior, permite observar dinámicas de aprendizaje, de apropiación, de circulación de conocimientos y de innovación social, no exentas de ries- 
gos ambientales y económicos, que se manifiestan en nuevos usos de la zarzamora y que están abriendo nuevas posibilidades al sistema productivo local con una densidad de actores e instituciones que, de manera aún dispersa, empiezan a operar en el territorio debido a la diversificación de canales de comercialización y a una incipiente acción institucional.

El sistema agroalimentario de la mora en Colombia se caracteriza por la integración jerárquica que desde las políticas públicas se pretende implementar para consolidar su cadena de valor. De acuerdo a los objetivos de crecer hacia el mercado internacional, el Ministerio de Agricultura promueve las moras colombianas en los mercados de exportación y tiene convenios con organismos internacionales, como el Instituto Interamericano de Cooperación para la Agricultura (IICA), para impulsar la productividad y la calidad de la frutilla.

Hay que señalar que en la base de esta cadena se encuentra la unidad agrícola familiar y su limitada producción. En un ámbito intermedio, se ubican los proveedores de insumos y servicios (Tobasura y Ospina, 2011), las agrotiendas y empresas de transporte, de abonos y/o fertilizantes así como los proveedores de insumos agropecuarios, entre otros.

A diferencia de Los Reyes, donde los intermediarios pueden asesorar al productor o proporcionarle crédito, en Pasto no intervienen en el proceso productivo, pues se limitan a la compra y venta del producto. Además del intermediarismo, las dificultades que enfrentan los productores de Pasto son semejantes a las existentes a nivel nacional: las deficiencias en el transporte (Criollo, Santacruz, Cardona y Ruano, 2010), el crédito (Bedoya et. al., 2004) y la escasa o inexistente cadena de frío (Egea 2015).

Como actor interesado en el impulso de este sistema agroalimentario y la consolidación de redes de comercialización nacional e internacional, se distingue el gobierno nacional, acompañado del gobierno departamental e incluso del municipal. La política pública pretende apoyarse en las asociaciones de productores porque podrían ser un actor clave en la generación de valor agregado. Desde 2017, ASOHOFRUCOL se encuentra explorando la posibilidad de "exportar mora congelada, debido a que la organización ya ha enviado muestras de su producto a otros países como Panamá y Estados Unidos" (ASOHOFRUCOL 2017).

Pero al ser un producto tradicional identificado con la agricultura familiar y con dificultades para su con- servación en anaquel, más allá de explorar el mercado de la mora congelada, las asociaciones de fruticultores, relacionadas con los grandes propietarios, no muestran un especial interés en esta fruta. Los gobiernos central y departamental, por el contrario, se han empeñado en desarrollar la cadena territorial de la mora no sólo por el interés de construir cadenas nacionales e internacionales de valor, sino por la creación de empleos en las áreas rurales; la generación de ingresos permanentes para las familias campesinas; la reconversión de los cultivos ilícitos y, por último, para implementar políticas de inclusión.

Por estas circunstancias, se ha dado énfasis a las políticas participativas en cuyo marco general se busca la consolidación del sistema agroalimentario local de la mora. Los gobiernos departamental y municipal vienen realizando esfuerzos para impulsar el desarrollo territorial, aprovechando la capacidad histórica de esta región para impulsar "la planeación y el presupuesto participativo, los planes de vida comunitarios, las políticas públicas de género y juventud, la erradicación voluntaria de cultivos de uso ilícito, entre otras" (Gobernación de Nariño 2016).

Sin embargo, a pesar de estos propósitos declarativos, existe la percepción, entre algunos actores, que la consolidación del sistema productivo de la mora y el desarrollo de redes de comercialización enfrentan graves dificultades, entre las cuales cabe mencionar: 1) las características mismas de la frutilla; 2 ) el intermediarismo; y 3) el cambio en las políticas públicas, que a partir de 2015 ha limitado los apoyos para su desarrollo.

En cuanto a la primera cuestión, resalta la rápida senescencia del fruto. La mora debe recolectarse en la tarde y venderse al intermediario a primeras horas de la mañana siguiente para ser trasladada a los mercados. De otra manera, la fruta se deshidrata y pierde valor.

Según las informaciones de los entrevistados, un predio promedio de $2000 \mathrm{~m}^{2}$, contiene aproximadamente $\mathbf{4 0 0}$ matas de mora, de las cuales cosechan entre 18 y 25 canastas semanales. A pie de finca, el productor vende a 3 US Dollars la canasta en promedio, pero el intermediario la revende en 5 US Dollars. Por esta razón, algunos productores organizados como los de "Agromoro" de Santa Clara, plantean potenciar las características del territorio, diversificando la oferta de productos territoriales. Para lograr su objetivo, demandan apoyos para la transformación de la frutilla, la promoción turística y la vinculación la mora con la oferta gastronómica. Como se puede 
notar, esta acción colectiva, dirigida por políticas públicas adecuadas, responde a la necesidad de construir sistemas agroalimentarios locales con una oferta diversificada, en la cual la mora es parte de una canasta de bienes y servicios territoriales, de acuerdo al concepto explorado por Pecqueur (2001), valorizando de este modo los productos identificados con el territorio.

Con relación al cambio en las políticas de apoyo, los productores entrevistados coincidieron en señalar que en el 2012 recibieron asistencia técnica y ayudas en especie, como semillas e insumos para incrementar la producción de mora, con lo que creció el área sembrada y cosechada. Sin embargo, el precio cayó $y$, por ende, la ganancia. El incremento finalmente fue sólo temporal debido a que, si bien el cultivo de mora es permanente, su mantenimiento demanda inversión constante de tiempo e insumos. Asimismo, señalaron que se les ofreció asistencia técnica, pero no se dio seguimiento. Algunos actores acusan, además, que los escasos apoyos se han canalizado a otros municipios por criterios políticos (Enrique C., dirigente de ASOHOFRUCOL, Comunicación personal, 21 de junio, 2017).

La figura 3 muestra una coordinación multinivel menos compleja que en el sistema de Los Reyes, Michoacán.

FIGURA 3

INTERACCIONES MULTINIVEL EN EL SISTEMA PRODUCTIVO DE MORA, COLOMBIA

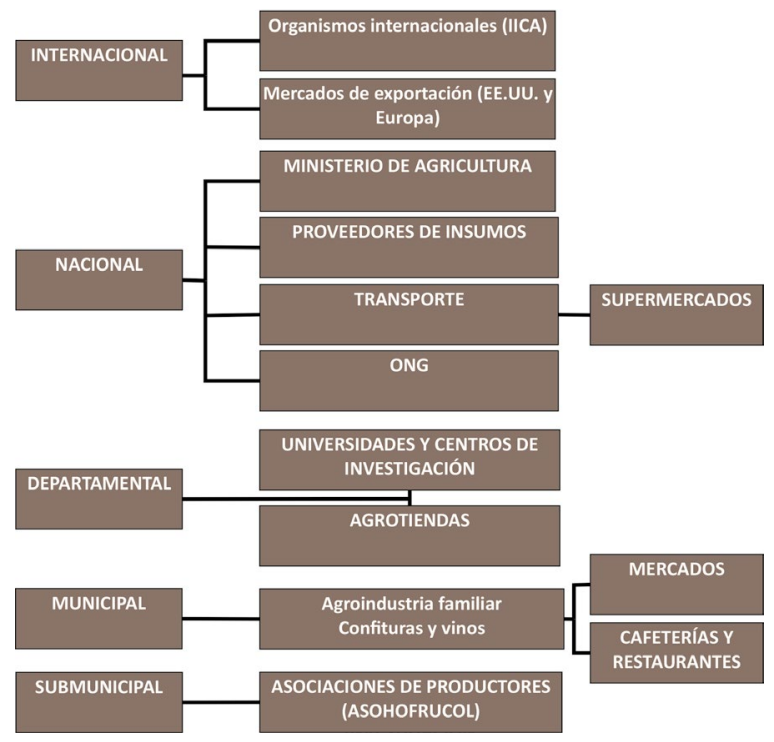

Fuente: Elaboración propia.

\section{Tensiones y conflictos ambientales}

En Los Reyes, el paisaje que aún hace veinte años estaba dominado por los cultivos de caña de azúcar, ahora se ha transformado por el monocultivo de la zarzamora (Thiébaut, 2012). La dinámica de los campos zarzamoreros es dictada por las buenas prácticas agrícolas que han impuesto las empresas agroexportadoras. Estas prácticas se relacionan con el tratamiento de los suelos, el agua y la aplicación de agroquímicos potencialmente peligrosos para la salud.

El plástico es utilizado en prácticamente todo el proceso productivo, pero es particularmente visible en el acolchado del suelo, la construcción de macrotúneles y sistemas de riego por goteo. El acolchado se utiliza con el objetivo de retener humedad y controlar las plagas; los macrotúneles para proteger la planta y el equipo de conducción del sistema de riego por goteo para racionalizar el uso del agua. Para los asistentes al taller FODA, además de los plásticos, los esquilmos de las plantas tampoco tienen una adecuada disposición, pues son colocados en los límites de las parcelas, convirtiéndose en hospederos de la drosophila suzukii, la cual ataca especialmente los cultivos de zarzamora y otras berries. Para los jornaleros, además, representa un problema de salud, pues se ven obligados a fumigar y no disponen del equipo adecuado.

En Los Reyes, el agua es un recurso abundante y de buena calidad que fluye de manantiales, pero en su recorrido por barrancas y ríos se contamina al mezclarse con las aguas residuales de los centros urbanos y de campos agrícolas (CONAGUA, s.f). Esta situación ha ocasionado que los grandes productores y comercializadores de frutillas exijan agua proveniente del acuífero. El acceso al agua limpia proporcionada por pozos tecnificados ha marcado tensiones y conflictos que se han resuelto a favor de los agricultores vinculados a las agroexportadoras.

En el municipio de Pasto, los riesgos ambientales derivan de la creciente contaminación del agua, debido a las descargas residuales y a la proliferación de lanchas motorizadas para el transporte de personas y mercancías. Según uno de los informantes, se necesita un reordenamiento de las quebradas (arroyos de poco caudal), pues se encuentran contaminadas por el aceite de las lanchas, lo cual afecta al río principal y al lago (Sr. Bolívar, dirigente de Agromoro, comunicación personal, 23 de junio, 2017). Otros riesgos derivan de la pérdida de rentabilidad de los cultivos tradicionales, entre ellos, la mora, por lo cual 
mucha gente "se ha ido al carbón" (productor de mora de Castilla, comunicación personal, 24 de junio, 2017). Los carboneros se encuentran constituidos en una asociación que ha tenido conflictos con la Corporación Autónoma Regional de Integración de Nariño y Putumayo (CORPONARIÑO), la instancia encargada de ordenar el recurso hídrico en el territorio e impulsar las cadenas productivas en el departamento.

Otra de las debilidades ambientales expresadas por varios entrevistados (funcionarios, un líder de productores y académicos) es que, a pesar de ser un cultivo tradicional, la mora necesita un alto uso de insumos por su vulnerabilidad ante las plagas, así como la ausencia de buenas prácticas para garantizar la calidad e inocuidad (Enrique C., dirigente de ASOHOFRUCOL, comunicación personal, 21 de junio, 2017). Los insumos químicos han afectado la variedad nativa de la zona (mora de Castilla), razón por la cual ha sido sustituida por variedades híbridas como la fresa mora, que si bien tienen mayor productividad, demandan una mayor inversión.

Las fumigaciones aéreas con glifosato, utilizado para la erradicación de los cultivos ilícitos en el vecino departamento de Putumayo, han contaminado y afectado los cultivos en Pasto. De acuerdo a un productor, cuando se esparcía el herbicida, perjudicaba la raíz de la planta que "se empezaba a podrir" (productor de mora de Castilla, comunicación personal, 24 de junio, 2017).

\section{DisCuSIÓN Y CONCLUSIONES}

En esta contribución se han descrito y analizado dos modelos de gobernanza construidos a partir de la demanda global de berries, que ha impulsado el crecimiento del área cosechada de la zarzamora y de la mora, tanto en México como en Colombia. El advenimiento las frutillas como mercancías altamente valorizadas en el mercado internacional ha provocado un ordenamiento de los territorios en función de las cadenas globales de valor, sobre todo en México. Siguiendo a Kooiman (2003) y sus modelos de gobernanza, la intervención en la acción social de los territorios ha seguido un patrón claramente jerárquico por dos agentes externos: las empresas agroexportadoras y la gestión pública.

Según González et al. (2020), la irrupción de esas cadenas organizadas por empresas agroexportadoras trajeron aparejadas no sólo una forma de gobernanza jerárquica, sino la captura de los productores con mayor potencial mediante la agricultura por contrato, con reglas estrictas de buenas prácticas agrícolas (Manzo, 2015) a quienes ofrecen buenas condiciones para la comercialización, pero al mismo tiempo prohíben introducir cualquier innovación.

En el esquema de la gobernanza jerárquica, la gestión pública subordina a los productores mediante controles fitosanitarios y regulaciones estrictas que garantizan los derechos de propiedad de obtentores vegetales para las grandes corporaciones, como en el caso de Los Reyes, o a través de la distribución de plántulas y semillas híbridas, como en el caso de Pasto.

Los efectos ambientales y sociales de esta forma de gobernanza son diversos: en el caso de Los Reyes, se pueden observar cambios drásticos en el paisaje como producto de la reconversión agrícola, la formación de empresas de producción, transformación y servicios aglomeradas en torno a las grandes empresas agroexportadoras, a la manera de un cluster, así como el rentismo y el crecimiento de la población asalariada en el territorio, lo que plantea demandas de mejores salarios, capacitación y seguridad social (Chollet, 2011; Paleta, 2012).

En el caso de Pasto, la gestión pública de los tres niveles de gobierno intentó, hasta 2015, impulsar la cadena de valor de la mora con base en estímulos a la economía campesina, cuyo resultado fue la comercialización del producto para obtener un ingreso complementario, pero mermado por el elevado uso de productos químicos y el intermediarismo.

Con respecto a los efectos ambientales, Sandoval y Ospina (2011) encontraron diferencias sustanciales entre el sistema campesino tradicional de Colombia y el empresarial de México. Para ellos, este último tiene ventajas en cuanto a la integración en el mercado, pero el modelo campesino tiene un manejo del medio ambiente más adecuado. No obstante, en esta investigación se muestra que ambos sistemas tienen tensiones ambientales por la aplicación de insumos químicos, la contaminación del agua y la sustitución de las especies nativas, en el caso de Pasto. Estas tensiones han derivado hacia otros conflictos socioambientales como el que sostienen CORPONARIÑO y los carboneros o las afectaciones que han provocado las fumigaciones de cultivos ilegales con glifosato.

Ambos casos de estudio demuestran el funcionamiento de la agricultura y de las áreas rurales en 
América Latina con la formación de una economía empresarial agroexportadora, por un lado, y el funcionamiento de la unidad campesina, por el otro. Desde este punto de vista, ambas formas de economía parecen irreductibles. En los casos estudiados, la intervención jerárquica en el territorio de los agentes privados o públicos pareciera dejar poco margen para pensar en alternativas de desarrollo. No obstante, como hemos visto aquí, en la medida que se desarrolla el sistema productivo, las reglas del juego se matizan o resignifican entre los actores locales por medio de procesos de negociación y adaptación ante la intervención de los agentes externos.

En el caso de los Reyes, cabe señalar la existencia de actores cuyo dinamismo los enmarca en un sistema productivo local en desarrollo: la difusión de las variedades que han sido liberadas por las agroexportadoras, como la Tupy; el aprendizaje del proceso productivo y de la transformación del producto para elaborar vinos, mermeladas, panes o postres; la difusión de cadenas de frío y el aprovechamiento de los canales incipientes de comercialización para el mercado interno, aunado a un impulso institucional y social de la innovación.

En Pasto, la inconsistencia de la acción pública y el intermediarismo han llevado a conservar la mora en el sistema de policultivo como una estrategia de seguridad alimentaria y de venta para generar ingresos permanentes. Para otros agricultores, las dificultades les han llevado a elaborar una oferta incipiente de servicios a la población urbana, en la cual la mora se integra en otras actividades como el turismo, la gastronomía y otros bienes ambientales, lo que marca la pauta para la elaboración de una canasta de bienes y servicios territoriales (Pecqueur, 2001) y por nuevas formas de coordinación con cierto grado de autonomía respecto a la intervención pública, tal y como lo están desarrollando algunos productores individuales y asociaciones como "Agromoro".

Frente a las fallas del mercado y de la gestión pública para generar un sistema de reglas equitativo, vale la pena señalar que las transformaciones en los sistemas de gobernanza jerárquicos abren tendencialmente el camino a formas descentralizadas o relacionales de coordinación y a formas híbridas con la participación de la gestión pública, las empresas y asociaciones de productores. Con la investigación en su estado actual, aún no está claro si estas formas de coordinación podrán consolidarse en un futuro cercano, pero sí es posible afirmar que se presentan tendencias que llevan al desarrollo de los SIAL, las cuales se manifiestan de múltiples maneras: el aprovechamiento de semillas y plantas que han dejado de ser aptas para la exportación, el trabajo para abrir canales de comercialización o las estrategias de promoción del territorio.

En este sentido, el estudio de las formas de gobernanza en diferentes contextos es útil para el conocimiento de los actores y las posibilidades de desarrollo. Tobasura y Ospina (2011) reconocieron esta necesidad para el caso de Caldas, cuando se preguntaron si el enfoque de la gobernanza en las cadenas de valor era relevante para inducir la formación de los SIAL. La pregunta actual es si, frente al dominio de las agroexportadoras, su poder tecnológico y su capacidad para deslocalizar la producción, los SIAL podrán anclar el producto al territorio y construir su autonomía o serán formaciones subordinadas y destinadas a producir la zarzamora o la mora con riesgos ambientales y con una calidad inferior.

En suma, el conocimiento de los modelos de gobernanza es de interés para la definición de la agenda y el diseño de políticas públicas dirigidas a la formación de SIAL, con la cooperación de los actores sociales e institucionales, con modelos de gobernanza descentralizada y oferta de servicios territoriales. En sus propias trayectorias de desarrollo, la interacción social, dentro de los sistemas productivos analizados, busca valorarizar el producto de acuerdo a las características particulares de cada uno de los territorios.

\section{RECONOCIMIENTOS}

Este artículo es resultado del proyecto UNAM-PAPIIT IN303117. Nuestro agradecimiento a los productores, jornaleros, funcionarios y académicos quienes nos han proporcionado las facilidades para realizar este trabajo. A Anahí Campos Tenango, Mariana Huerta Vázquez y a Yunuen Guadalupe Guerra Villa por su participación en el trabajo de campo, así como a José Norwin por su apoyo técnico. 


\section{NOTAS}

1 En lo sucesivo, nos referimos a la Rubus fruticosus como zarzamora y a la Rubus glaucus Benth como mora.

2 Los nombres de los informantes fueron cambiados en la mayoría de los casos, pues así nos lo

\section{REFERENCIAS BIBLIOGRÁFICAS}

Aguilar, D. (5 de mayo de 2018). Zarzamora mexicana tupy sin futuro de exportación.En Valencia, Jess, Historias del Valle Esmeralda de Los Reyes, Michoacán y sus alrededores [Blog]. Recuperado de: https://xesvalencia.blogspot.com/2018/05/ zarzamora-mexicana-tupy-sin-futuro-de.html

Aguilar, L. (2006). Gobernanza y gestión pública (Primera ed.). México: Fondo de Cultura Económica.

Alwang, J., Barrera, V., Andrango, G., Dominguez, J., Martinez, A., Escudero, L., y Montufar, C. (2019). Value-chains in the Andes: Upgrading for Ecuador's blackberry producers. Journal of Agricultural Economics, 70(3), 705-730. doi:https:// doi.org/10.1111/1477-9552.12329

Asociación Hortofrutícola de Colombia, ASOHOFRUCOL. (2017). Asofrunidos le apuesta a una producción ambiental y económicamente sostenible. Frutas y Hortalizas. Revista de La Asociación Hortofruticola de Colombia, ASOHOFRUCOL, 51, 1820. Recuperado de: http://www.asohofrucol. com.co/archivos/Revista/Revista51.pdf

Bedoya, F. et al. (2004). Cultura organizativa y participativa en el ordenamiento y manejo de microcuencas de los corregimientos de genoy, obonuco y buesaquillo. Municipio de Pasto. San Juan Pasto, Colombia: Universidad de Nariño. Recuperado de: https://drive.google.com/file/d/0B4me103 pWFMHZE1CdGpjTDZkWnc/view

Benavides, M., Tarlé, T., y Galbiatti J. (2009). Caracterización y clasificación de la red hidrográfica de la cuenca del río Bobo, departamento de Nariño - Colombia. Revista Ingeniería e investigación. 20(3), 97-101. Recuperado de https://revistas. unal.edu.co/index.php/ingeinv/article/ view/15190/15984 solicitaron los entrevistados, sobre todo en Michoacán.

3 El US Dollar tenía una equivalencia de 20 pesos mexicanos en promedio y de 3000 pesos colombianos en el momento de la realización del estudio.

Centro de Innovación y Desarrollo Agroalimentario de Michoacán A.C. (CIDAM). https://www.cidam.org/

Chollet, D. L. (2011). Renegotiating gender and class in the berry fields of Michoacán, Mexico. Dialectical Anthropology, 35(2), 147-169. doi:https:// doi.org/10.1007/s10624-010-9183-9

Comisión Económica para América Latina y el Caribe, CEPAL. (2016). Encadenamientos productivos y circuitos cortos: Innovaciones en esquemas de producción y comercialización para la agricultura familiar. Análisis de la experiencia internacional y latinoamericana. Santiago de Chile: CEPAL. Recuperado de: https://repositorio.cepal.org/ bitstream/handle/11362/40688/1/ S1600739_es.pdf

Comisión Nacional del Agua (CONAGUA), Gobierno de México (s.f). Programas contra contingencias hidráulicas por organismos de cuenca y para las principales ciudades del país (etapa 1). Subdirección General Técnica Gerencia De Aguas Superficiales e Ingeniería de Ríos.

Consejo Nacional de Política Económica y Social (CONPES), República de Colombia, Departamento Nacional de PIneación. Documento 3811(2014). Políticas y estrategias para el desarrollo agropecuario del departamento de Nariño. Bogotá: Gobierno de Colombia. Recuperado de: https:// colaboracion.dnp.gov.co/CDT/Conpes/ Econ\%C3\%B3micos/3811.pdf

Criollo, L., Santacruz, M., Cardona, J. y Ruano, V. (2010). Análisis del subsector agrícola de Nariño. 2000-2005. San Juan Pasto, Nariño, Colombia: Centro de Estudios del Desarrollo Regional y Empresarial (CEDRE). Recuperado de: http://cedre. udenar.edu.co/portfolio/analisisdel-subsector-agricola-de-narino-2000-2005/ 
Daigle, J., Michelle, N., Ranco, D., y Emery, M. (2019). Traditional lifeways and storytelling: Tools for adaptation and resilience to ecosystem change. Human Ecology: an Interdisciplinary Journal, 47(5), 777-784.doi: https://doi.org/10.1007/ s10745-019-00113-8

Departamento Administrativo Nacional de Estadística, DANE. Gobierno de Colombia (2019). https:// w w w. dan e.gov. co/index.ph p / estadisticas-por-tema

Egea, L. (2015). Desarrollo de una propuesta de mejoramiento de productividad y competitividad en cadenas hortofrutícolas a partir de la evaluación tecnológica de la cadena de frío. Caso de estudio: Mora de castilla y papa criolla (Tesis de Maestría). Recuperada de https://repositorio.unal.edu.co/ handle/unal/56238

Fideicomisos Institucionales en relación con la Agricultura, FIRA, Gobierno de México (2016). Panorama agroalimentario. Berries 2016. México: FIRA. Recuperado de: https://www.gob.mx/cms/ uploads/attachment/file/200633/Panorama_ Agroalimentario_Berries_2016.pdf

Flyvbjerg, B. (2001). Making social science matter: Why social inquiry fails and how it can succeed again. Cambridge: Cambridge University Press.

Food and Agriculture Organization of the United Nations, FAO. (2019). FAOSTAT. http://www.fao.org/ faostat/en/\#home

Giacomini, C., y Mancini, M. (2015). Organisation as a key factor in Localised Agri-Food Systems (LAFS). Bio-based and Applied Economics, 4(1), temp1temp16. doi: https://doi.org/10.13128/BAE15088

Giménez, G., y Heau, C. (2014). El problema de la generalización en los estudios de caso. En C. Oemichen (Ed.), La etnografía y el trabajo de campo en las ciencias sociales (pp. 347-364). México: UNAM-IIA.

Gobernación de Nariño, Gobierno de Colombia (2016). Plan participativo de desarrollo departamental. Nariño, corazón del mundo. 2016-2019 Gobernación de Nariño:Colombia. Recuperado de http://www.2016-2019.narino.gov.co/inicio/ in d ex . ph p / g oberna cion / p l a n - d e desarrollo/354-plan-de-desarrollo-departamentalnarino-corazon-del-mundo-2016-2019
Gobierno de Colombia (2020). http://www.ideam. gov.co/web/tiempo-y-clima

González, M., Santoyo, V., Arana, J., y Muñoz, M. (2020). The insertion of Mexico into global value chain of berries. World Development Perspectives, 20(100240). doi: https://doi.org/10.1016/j. wdp.2020.100240

Henao, A., y Tobasura, I. (2018). Enfoques de extensión rural para el desarrollo de productores de mora en el departamento de Caldas. Ciencia y Agricultura, 15(2), 25-38. doi: https://doi. org/10.19053/01228420.v15.n2.2018.8393

Hoffmann, O., y Agudelo, C. (1998). Políticas agrarias, reformas del Estado y afiliaciones identitarias: Una comparación entre Colombia y México. Revista Mexicana de Sociología, 60(4), 99-123. doi: https://doi.org/10.2307/3541333

Ibarra, L., Romero, N., Jaime, R., y Hurtado, B.(2013). Estudio de factibilidad para la comercializacion de zarzamora en mercados internacionales. Revista Internacional Administracion y Finanzas, 6(2), 57-71. Recuperado de https://papers.ssrn. com/sol3/papers.cfm?abstract_id=2156449

Infante, Z., y Trueba R. (2014). Innovaciones agrícolas para impulsar el desarrollo sustentable en los Reyes Michoacán: el caso de la Zarzamora. Revista DELOS: Desarrollo Local Sostenible, (20). Recuperado de http://www.eumed.net/rev/delos/20/ michoacan.html

Instituto Nacional de Estadística y Geografía (INEGI). (2009). Prontuario de información geográfica municipal de los Estados Unidos Mexicanos. Los Reyes, Michoacán de Ocampo. Recuperado de http://www3.inegi.org.mx/contenidos/app/ mexicocifras/datos_geograficos/16/16075.pdf

Kooiman, J. (2003). Governing as governance. LondonThousand Oaks-New Dehli: Sage publications.

Manzo M. (2015). El análisis de la cadena de valor como fuente de ventajas competitivas en las empresas exportadoras de zarzamora en México. Red Internacional de Investigadores en Competitividad 9(1) 1123-1143Recuperado de https:// riico.net/index.php/riico/article/view/63

Merchand, M. (2013). La trasnacionalización de la agricultura de exportación en México. Geografia Em Questão, 6(2), 235-261. Recuperado de http://e-revista.unioeste.br/index.php/ geoemquestao/article/download/5633/6386 
Ministerio de Agricultura, Gobierno de Colombia. (2015). Cadena Productiva Nacional de la Mora. Indicadores de apoyo. Marzo, sle. Recuperado de https://sioc.minagricultura.gov.co/Mora/ Documentos/2014-10-31\%20Cifras\%20 Sectoriales.pdf

Ministerio de Agricultura. Agronet. Gobierno de Colombia. (2020). https://www.agronet.gov.co/ Paginas/ProduccionNacionalProducto.aspx

Morales, Y., y Cantillo, E. (2017): Red de comercialización de zarzamora en Los Reyes, Michoacán. Commercium PLUS, 2(1), 29-54.

Morales, Y., Cantillo, E., y Colín, R. (2019). Indicadores de integración en la red de comercialización de la zarzamora en el municipiio de los reyes, michoacán. Economía y Sociedad. Nueva Época, 38(22), 53-70. Recuperado de http://www. economiaysociedad.umich.mx/ojs3/index.php/ ecosoc/article/view/9/5

Moreno, M., Villareal, D., Lagos, C., Ordoñez, H., y Criollo, H. (2011). Caracterización"in situ" de genotipos silvestres y cultivados de mora rubus spp en el municipio de pasto. Revista De Ciencias Agrícolas, XXVIII(2), 109-128. Recuperado de https://dialnet.unirioja.es/descarga/articulo/ 5104090.pdf

Muñoz, M., y Juárez, M. (1995). El mercado mundial de la fresa y la zarzamora. México: Universidad Autónoma Chapingo-Aserca-CIESTAAM

Paleta, G. (2012) Territorios y ruralidades: Jornaleros agrícolas en el cultivo de zarzamora en el valle de los Reyes, Michoacán, México. Revista de Antropología Experimental, 12(2012), 17-28. Recuperado de http://revista.ujaen.es/huesped/rae/ articulos2012/02paleta12.pdf

Pecqueur, B. (2001). Qualité et développement territorial: I'hypothèse du panier de biens et de services territorialisés. Économie Rurale, 261(1), 37-49. doi: https://doi.org/10.3406/ecoru.2001.5217

Porras, F. (2019). Gobernanza y gobernanza territorial; aportaciones al debate. Cuadernos Americanos, 169(3), 15-38. Recuperado de http://www. cialc.unam.mx/cuadamer/textos/ca169-15.pdf

Programa de las Naciones Unidas para el Desarrollo, PNUD (s.f). Sector agroalimentario. Oportunidades de inclusión productiva para poblaciones en situación de pobreza y vulnerabilidad de Pasto. Bogotá: PNUD. Recuperado de https://info.undp.
org/docs/pdc/Documents/COL/00058568_ Estudio\%20Perfiles\%200cupacionales\%20Pasto.pdf

Sánchez, G. (2008). La Red de Valor de la zarzamora. El clúster de Los Reyes, Michoacán, un ejemplo de reconversión competitiva. Morelia, Michoacán: Fundación Produce Michoacán.

Sandoval, A. (2016). ¿Gobernanza?: Adaptaciones y organización en la producción de berries en Michoacán. En Torres, G. (coord), Territorios en movimiento. Sistemas agroalimentarios localizados. Innovación y gobernanza (pp. 273-296). México: Bonilla Artigas Editores.

Sandoval, A. (2019). "Gobernanza y procesos socioterritoriales: el caso de las frutillas en Michoacán". En: Cuadernos Americanos, 169. pp. 119139. Recuperado de http://www.cialc.unam.mx/ cuadamer/textos/ca169-119.pdf

Sandoval A., y Ospina P. (2011). Sustentabilidad ambiental en el manejo del agua y del suelo en la producción de berries. Los casos de México y Colombia. Ambiente y Desarrollo, 15(28), 99-121. Recuperado de https://dialnet.unirioja.es/ servlet/articulo?codigo $=3937720$

Sanz, J., y Muchnik, J. (2016). Geographies of origin and proximity: Approaches to local agro-food systems. Culture \& History Digital Journal, 5(1), e002. doi: http://dx.doi.org/10.3989/chdj. 2016.002

Schmeda, G., Jiménez, F., Theoduloz, C., y Ladio, A. (2019). Patagonian berries as native food and medicine. Journal of Ethnopharmacology, 241, 111979-111979. doi: https://doi.org/10.1016/j. jep.2019.111979

Servicio de Información Agroalimentaria y Pesquera (SIAP). Gobierno de México (2020). Recuperado de https://www.gob.mx/siap/

Servicios Informativos y Publicitarios del Sureste Mexicano, SIPSE. (2014). La zarzamora, el nuevo oro rojo de mlchoacán. Recuperado de https:// sipse.com/mexico/zarzamora-mexicana-frutomundo-michoacan-exportacion-112481.html

Servicio Nacional de Sanidad, Inocuidad y Calidad Agroalimentaria (SENASICA), Gobierno de México, (2019). Huertos autorizados para exportación. Recuperado de https://www.gob.mx/senasica/ documentos/unidades-de-produccion

Sistema de Información de Precios y Abastecimiento del Secto Agropecuario, (SIPSA), Ministerio de 
Agricultura, Gobierno de Colombia (2013, noviembre). El cultivo de mora de castilla (rubus glaucus benth) frutal de clima frío moderado, con propiedades curativas para la salud humana. Boletín Mensual. Insumos y Factores Asociados a La Producción Agropecuaria, 1-7. Recuperado de https://docplayer.es/16372857-El-cultivo-de-lamora-de-castilla-rubus-glucus-benth-frutal-declima-frio-moderado-con-propiedades-curativaspara-la-salud-humana.html

Thiébaut, V. (2009). De la caña a la zarzamora: el Valle de Los Reyes en proceso de transformación. En Téllez V. y García S. (Eds.) Estudios Michoacanos XIII, El Colegio de Michoacán, Colección Estudios, Zamora, (pp. 13-38). Zamora, México: El Colegio de Michoacán

Thiébaut, V. (2011). Paisajes rurales y cultivos de exportación. Valle de los Reyes, Michoacán. Trayectorias, 13(32), 52-70. Recuperado de http://www. trayectorias.uanl.mx/32/index.htm

Thiébaut, V. (2012). La transformation des paysages agraires mexicains, cultures de la canne à sucre et de la mûre dans la vallée de Los Reyes, Michoacán. Cibergeo: European Journal of Geography, (626), 1-24. doi: https://doi.org/10.4000/ cybergeo. 25615

Tobasura, I.,y Ospina, C. (2011). Cadena de la mora en Caldas, Colombia: Beneficios e impactos. Eutopía. Revista de Desarrollo Económico y Territorial, 2 (Octubre), 81-100. doi: https://doi. org/10.17141/eutopia.2.2010.1030
Torre, A., y Traversac, J. (Eds.). (2011). Territorial governance. Local development, rural areas and agrofood systems. New York: Springer. doi: https://doi.org/10.1007/978-3-7908-2422-3

Torres, G., y Ramos, A. (2008). Gobernanza y territorios. Notas para la implementación de políticas para el desarrollo. Revista Mexicana de Ciencias Políticas y Sociales, L (203), 75-95. doi: http:// dx.doi.org/10.22201/fcpys. 2448492 xe. 2008.203.41994

Torres, G., y Sanz, J. (2018). Territorial governance. A comparative research of local agro-food systems in Mexico. Agriculture, 8(18), 1-15. doi: https:// doi.org/10.3390/agriculture8020018

Trueba, R., Infante, Z., \& Ayala, D. (2018). Sistemas agroalimentarios localizados: El caso del sector productivo de la zarzamora en Los Reyes, Michoacán. En P. Ortega, Z., Infante y C. Ortiz (Eds.), Transferencia de conocimiento y sistemas de innovación para el desarrollo sustentable pp. 43-56. Guadalajara, México: CENID. Recuperado de https://www.researchgate.net/publication/ 333994750_Sistemas_agroalimentarios_localizados_ el_caso_del_sector_productivo_de_la_zarzamora_ en_Los_Reyes_Michoacan

United States Department of Agriculture USDA, Economic Research Service. (2019). Recuperado de https://www.ers.usda.gov/ 